\title{
Developer Design of Hybrid Plasmonic Nano Patch Antenna with Metal Insulator Metal Multilayer Construction
}

\author{
Raed M. Shaaban $\mathbb{D}^{1},{ }^{1}$ A. Mudhafer $\mathbb{D}^{2},{ }^{2}$ and Ra'ed Malallah ${ }^{1}{ }^{1}$ \\ ${ }^{1}$ Physics Department, Faculty of Science, University of Basrah, Garmat Ali, Basrah, Iraq \\ ${ }^{2}$ Chemical and Petrochemical Technical Engineering Department, Southern Technical University, Basrah, Iraq \\ Correspondence should be addressed to A. Mudhafer; a.mudhafer@stu.edu.iq
}

Received 21 June 2019; Accepted 3 October 2019; Published 19 November 2019

Academic Editor: Zhiyong Xu

Copyright (c) 2019 Raed M. Shaaban et al. This is an open access article distributed under the Creative Commons Attribution License, which permits unrestricted use, distribution, and reproduction in any medium, provided the original work is properly cited.

\begin{abstract}
Hybrid plasmonic nanopatch antenna with metal-insulator-metal (HMIM) multilayer has been investigated for operation at the frequency of 125-250 THz using the finite element method (FEM) implemented in Ansoft High Frequency Structure Simulator (HFSS). The proposed antenna exhibits a wide bandwidth of $49.5 \mathrm{THz}(151.5 \mathrm{THz}-201 \mathrm{THz})$ for the slots thicknesses $W_{\mathrm{g}}=50 \mathrm{~nm}$ and $W_{\mathrm{s}}=100 \mathrm{~nm}$ and dual bandwidth for $W_{\mathrm{s}}=20 \mathrm{~nm}$. The obtained results show the input impedance of $50.3 \Omega$ input resistance (real part) and $2.3 \Omega$ reactance (imaginary part) occurring at (near) the operation frequency. The maximum gain of $23.98 \mathrm{~dB}$ has been observed for resonant frequencies of $176 \mathrm{THz}$, and the maximum directivity remains above $6.73 \mathrm{~dB}$ and $7.46 \mathrm{~dB}$ at resonant frequencies of $170 \mathrm{THz}$ and $190 \mathrm{THz}$, respectively. Our proposed antenna performance is compared to previously reported designs. The copolar and cross-polar radiation patterns are simulated at different resonant frequencies of $160 \mathrm{THz}$ and $197 \mathrm{THz}$ for planes $\Phi=90^{\circ}$ and $\Phi=0^{\circ}$. The arrays of a proposed antenna are designed in one and two dimensions in order to appropriate high-gain applications.
\end{abstract}

\section{Introduction}

The topic of optical antennas has a historic background in previous years, but it has recently attached renewed interest for different reasons. One of the reasons is the availability of various routine nanoscale fabrication technologies that allow suitable sized structures to be made and explored as a way of harnessing nanoantennas [1-10]. A variety of nanoantennas have been investigated for different applications from light excitation or emission [11] to photovoltaics [12], scatters to redirect incident light [13], and optical wireless nanolink [14]. Nanoantennas have been proposed to enhance the efficiency of spectroscopy [15], sensing [16], and heat transfer [17]. The optical diffraction limit is a great obstacle to miniaturize applications in optical devices [18]. One approach to overcome this problem is utilizing the properties of surface plasmons which provide an effective way to solve it, in which the optical fields are highly confined beyond the diffraction limitation $[19,20]$. Essentially, surface plasmons are the collective resonant of electrons at the metal-dielectric interface and then the optical power is transformed into surface waves propagating along the interface [21]. Based on the above points, the plasmonic waveguides are suitable for either efficient transmission of optical signals or high confinement of optical fields, e.g., channel plasmons waveguides [22], metallic nanowires [23], nanoslots [24], and hybrid plasmonic waveguides [25]. The concept of plasmonic nanoantennas, as one of the most important achievements, was firstly designed for optical field enhancement. So far, several nanoantennas designs have been motivated from conventional microwave antennas, for instance, Vivaldi [9], rectangular and circular patch [8], grating antennas [26], Yagi-uda [27], dipoles [28], bowtie antennas [29], metallic nanoslit with cavity [30], etc. to achieve the aforesaid applications. However, mostly proposed antennas such as dipole, bowtie, and spiral are suitable for near-field applications only; their far-field radiation is very poor unlike the radio antennas, which deteriorates 


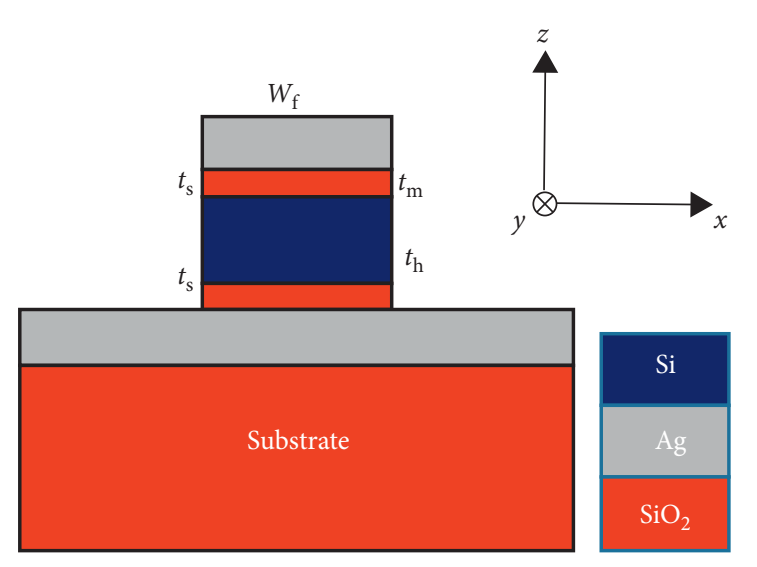

(a)

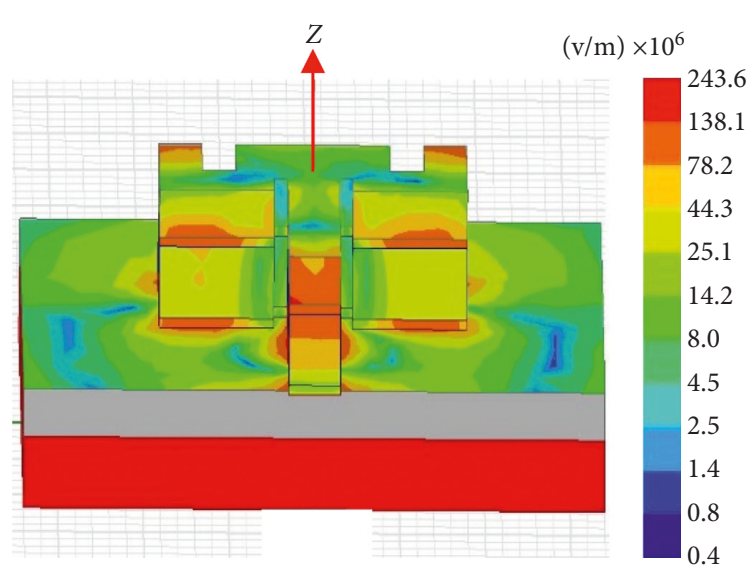

(b)

FIGURE 1: HMIM plasmonic feed waveguide. (a) Cross-sectional view. (b) 3D electric field view.

wireless interconnection capability. Contrary to this, patch antennas are apt for making a wireless link between two waveguide components due to its directional far-field pattern. Previous works are focused on the nanoantennas based on purely plasmonic. The authors did not pay more attention for the following two points: firstly, the purely plasmonic nanoantennas offer very poor efficiency and are not practically realizable due to excessive ohmic losses [31]. Secondly, the antenna designed for optical communication purposes must be able to receive an optical in-plane signal and radiate it to free space and through reciprocity and to receive the signal from free space and transmit in-plane signal. The far-field specifications of the antennas must be optimized for angle and maximum directivity. In this paper, the hybrid metal-insulator-metal (HMIM) plasmonic rectangular patch nanoantenna is developed for operation at standard communication frequencies of $125 \mathrm{THz}-250 \mathrm{THz}$ with a suitable feeding. With respect to losses resulting from the propagation and confinement, the proposed antenna exploits the low loss properties and subwavelength mode confinement of hybrid plasmonic structures, thus having a higher bandwidth, gain, and directivity than plasmonic antennas $[8,10,32,33]$. Since proposed inset fed HMIM plasmonic nanoantenna is highly directive, it can provide an efficient optical wireless interconnection. Moreover, the device can be used to enhance the absorption enhancement in photodetectors, solar cells, optical sensing, and wireless energy harvesting. The organization of the present work is as follows: the simulation structures of the HMIM plasmonic waveguide feed and nanopatch antenna are outlined in Section 2. The obtained results are presented in Section 3. In Section 4, the research work is concluded.

\section{Simulation Structures and Methods}

The cross-sectional schematic diagram of the HMIM plasmonic waveguide feed is depicted in Figure 1, where a core layer of high dielectric constant material ( $\mathrm{Si}$ ) is sandwiched between a low dielectric constant material $\left(\mathrm{SiO}_{2}\right)$ (spacer) and a metal (Ag). The structure supports a guided plasmonic TM mode which is highly confined inside the material with high dielectric constant. Dispersive permittivity of sliver is $\varepsilon=\varepsilon^{\prime}-j \varepsilon^{\prime \prime}$, where $\varepsilon^{\prime}$ and $\varepsilon^{\prime \prime}$ are the real and imaginary parts, respectively.

In the HFSS model, $\varepsilon^{\prime}$ can be calculated according to the Drude model of silver [34]. The optimized dimensions of feed waveguide are set as follow: the waveguide width $W_{\mathrm{f}}=150 \mathrm{~nm}$, the waveguide length $L_{\mathrm{f}}=540 \mathrm{~nm}$, metal thickness $t_{\mathrm{m}}=100 \mathrm{~nm}$, spacer thickness $t_{\mathrm{s}}=20 \mathrm{~nm}$, and high dielectric thickness $t_{\mathrm{h}}=150 \mathrm{~nm}$. The schematic diagram of the HMIM plasmonic nanopatch antenna is shown in Figure 2, which is designed in a rectangular form where patch width and length are set as $W_{\mathrm{p}}=315$ and $L_{\mathrm{p}}=900 \mathrm{~nm}$, respectively. When the guided hybrid plasmonic mode enters to the antenna, it transforms to radiating mode due to the four slots created in the design: two slots on either side of the feeding waveguide and the other two on the corresponding side with lengths $L_{\mathrm{g}}=80 \mathrm{~nm}$ and $L_{\mathrm{s}}=180 \mathrm{~nm}$ and thicknesses $W_{\mathrm{g}}=50 \mathrm{~nm}$ and $W_{\mathrm{s}}=80 \mathrm{~nm}$.

\section{Simulation Results and Analysis}

The Ansoft High Frequency Structure Simulator (HFSS) based on finite element method (FEM) is used to numerically analyze the antenna parameters such as reflection, VSWR, gain, directivity, and radiation patterns. The simulations in $[5,27]$ are tested using HFSS, and results obtained are very promising, which authorizes the simulation process. The ratio of reflection coefficients for HMIM has been illustrated in Figures 3(a) and 3(b), as a function of operation frequency $(125 \mathrm{THz}-250 \mathrm{THz})$ with slots varying in thickness $W_{\mathrm{g}}$ and $W_{\mathrm{s}}$ at $10 \mathrm{THz}$ with a $-10 \mathrm{~dB}$ bandwidth of approximately $151.5 \mathrm{THz}-201 \mathrm{THz}$ or $49.5 \mathrm{THz}$ for $W_{\mathrm{g}}=40 \mathrm{~nm}$ (see Figure 3(a)) and dual bandwidth for $W_{s}=20 \mathrm{~nm}$ (see Figure 3(b)), which is covering the whole optical communication. Moreover, observed bandwidth is larger than that reported in the literature $[8,10,32,33]$. Resonance frequency and bandwidth (BW) are compared in Table 1 for all slots thicknesses. It is clear from the comparison that the perfect 


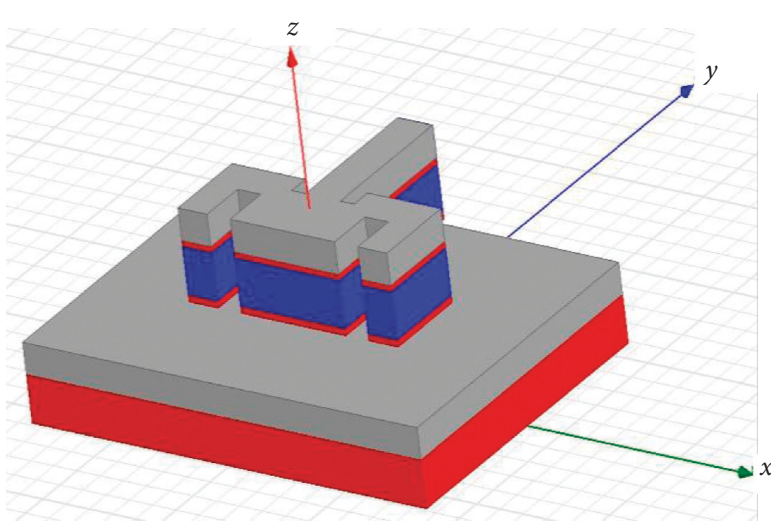

(a)

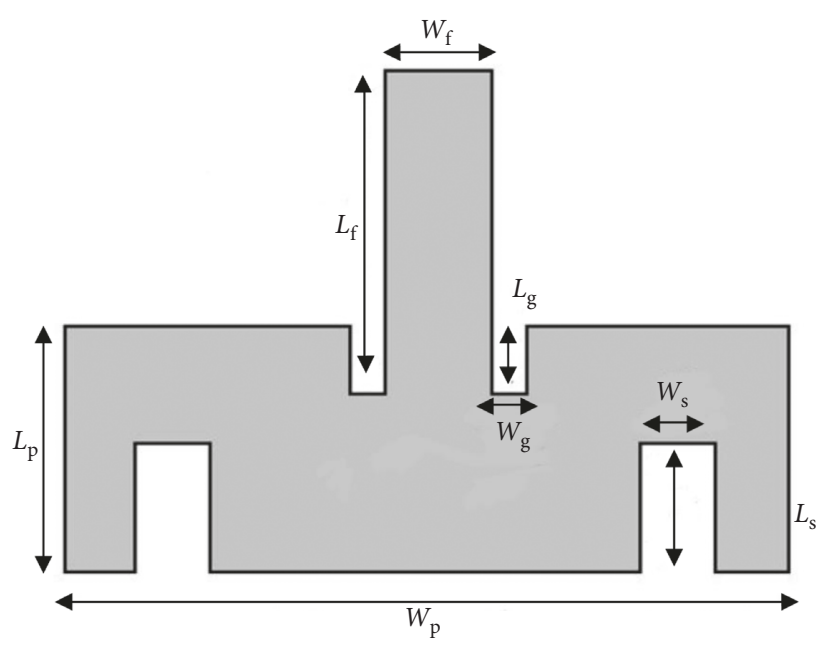

(b)

FIgURe 2: HMIM plasmonic rectangular nanopatch antenna with inset feed. (a) Perspective view. (b) Top view.

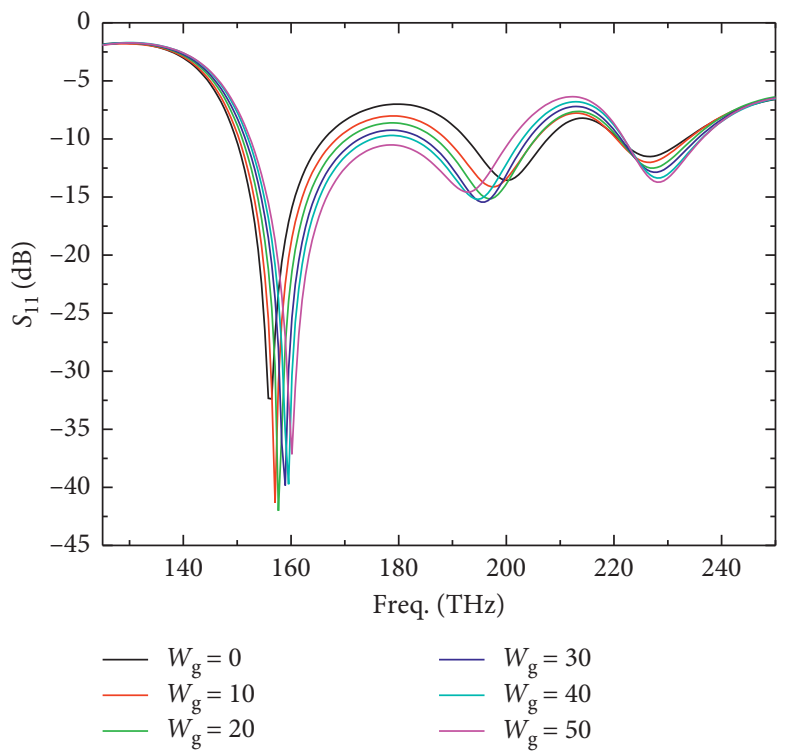

(a)

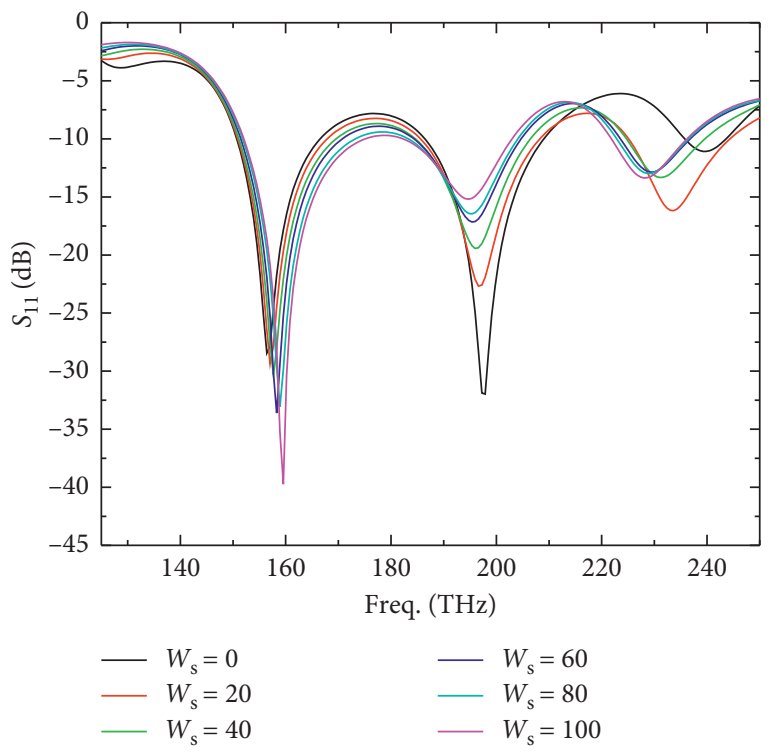

(b)

FIGURE 3: The ratio of reflected wave to the incident wave, $S_{11}$, versus operation frequency for different slots thicknesses. $(\mathrm{a}) W_{\mathrm{g}}=(0,10,20$, $30,40,50) \mathrm{nm}$. (b) $W_{\mathrm{s}}=(0,20,40,60,80,100) \mathrm{nm}$.

TABLE 1: Comparison at different slots thicknesses.

\begin{tabular}{|c|c|c|c|c|c|c|c|c|c|c|c|}
\hline$W_{\mathrm{g}}(\mathrm{nm})$ & \multicolumn{2}{|c|}{0} & \multicolumn{2}{|c|}{10} & \multicolumn{2}{|c|}{20} & \multicolumn{2}{|c|}{30} & \multicolumn{2}{|c|}{40} & 50 \\
\hline Freq. (THz) & \multicolumn{2}{|c|}{156.4} & \multicolumn{2}{|c|}{157} & \multicolumn{2}{|c|}{157.6} & \multicolumn{2}{|c|}{158.9} & \multicolumn{2}{|c|}{159.5} & 160.17 \\
\hline BW (THz) & \multicolumn{2}{|c|}{15.7} & \multicolumn{2}{|c|}{18.2} & \multicolumn{2}{|c|}{18.8} & \multicolumn{2}{|c|}{21.3} & \multicolumn{2}{|c|}{23.8} & 49.6 \\
\hline$W_{\mathrm{s}}(\mathrm{nm})$ & \multicolumn{2}{|c|}{0} & \multicolumn{2}{|c|}{20} & \multicolumn{2}{|c|}{40} & \multicolumn{2}{|c|}{60} & \multicolumn{2}{|c|}{80} & 100 \\
\hline Freq. $(\mathrm{THz})$ & 157 & 197.2 & 157.6 & 195.9 & 158.3 & 196 & 159.5 & 194.7 & 159.5 & 195.3 & 160.17 \\
\hline BW (THz) & 16.3 & 21.3 & 18.2 & 21.9 & 18.2 & 20.7 & 22.6 & 21.9 & 24.5 & 22 & 49.6 \\
\hline
\end{tabular}

resonance frequency is at $159.5 \mathrm{THz}$ for $W_{\mathrm{g}}=40 \mathrm{~nm}$ and at $197.8 \mathrm{THz}$ for $W_{\mathrm{s}}=100 \mathrm{~nm}$.

To precisely understand the antenna properties and the plasmonic effect, Figure 4(a) shows the simulated input impedance which consists of the resistance reactance of the proposed antenna. Input impedance of the proposed antenna shows $50.3 \Omega$ input resistance (real part) and $2.3 \Omega$ reactance (imaginary part) occurring at (near) the operating 


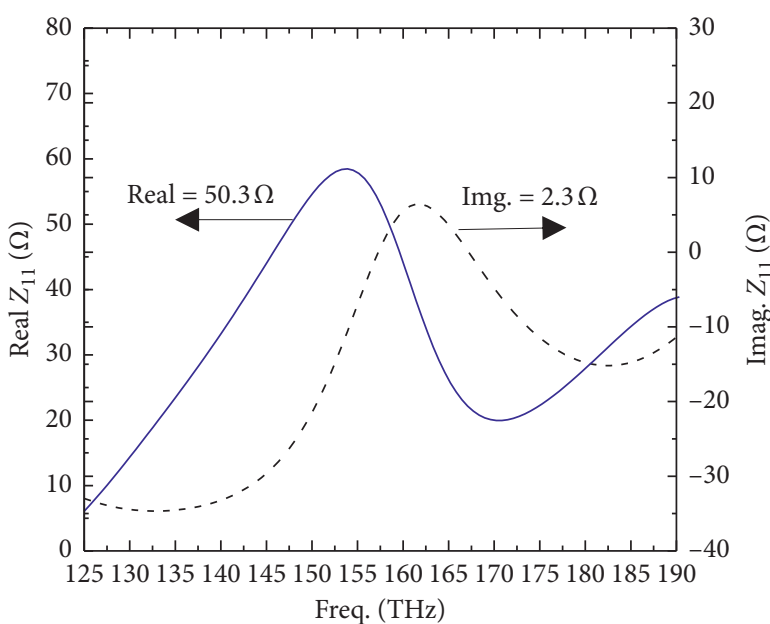

(a)

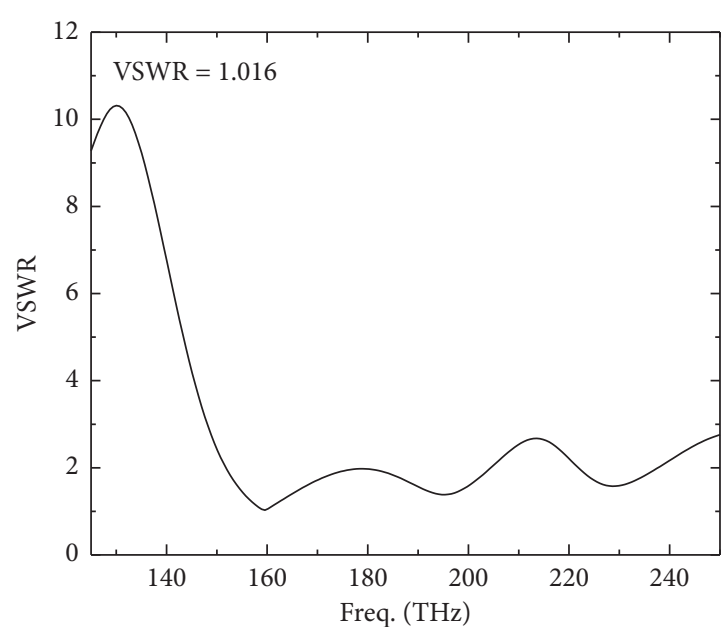

(b)

FIGURE 4: HMIM nanopatch antenna results for $W_{\mathrm{g}}=50 \mathrm{~nm}$ and $W_{\mathrm{s}}=80 \mathrm{~nm}$. (a) Real and imaginary impedance. (b) VSWR.

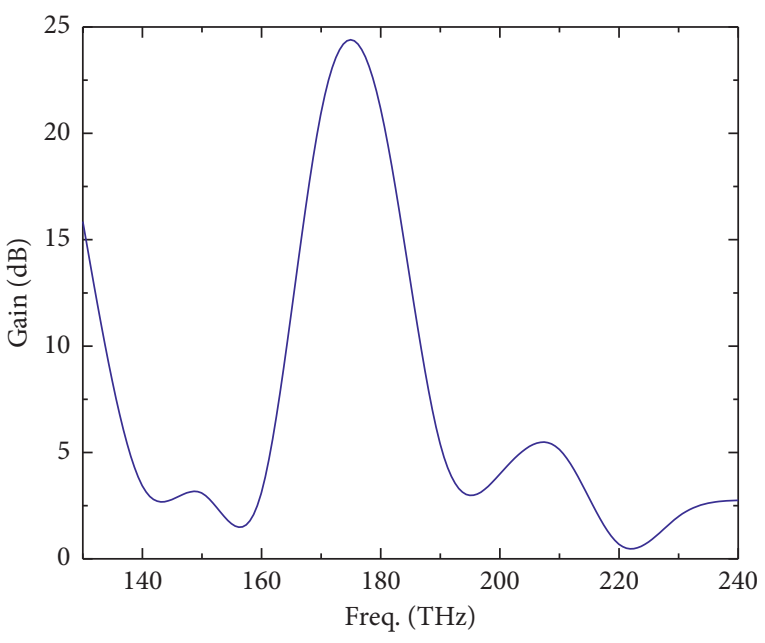

(a)

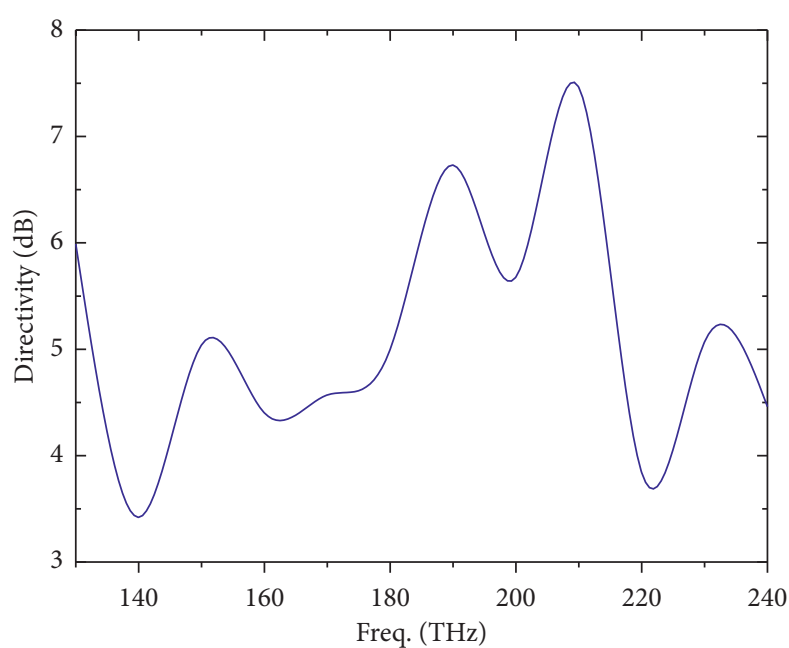

(b)

Figure 5: HMIM nanopatch antenna results for $W_{\mathrm{g}}=50 \mathrm{~nm}$ and $W_{\mathrm{s}}=80 \mathrm{~nm}$. (a) Gain radiation. (b) Directivity.

frequency. Perfectly, reactance (imaginary part) must be zero at resonance frequency, but here the given value is not zero due to plasmonic effect. Next, the Voltage Standing Wave Ratio (VSWR) efficiency, which is defined as the number of mismatches between an antenna and the feed line connection, has been shown in Figure 4(b).

In Figures 5(a) and 5(b), gain and directivity have been calculated, respectively. The gain of more than $23.98 \mathrm{~dB}$ has been observed for full frequencies band, as shown in Figure 5(a). The maximum directivity remains above $6.73 \mathrm{~dB}$ and $7.46 \mathrm{~dB}$ at resonant frequencies of $170 \mathrm{THz}$ and $190 \mathrm{THz}$, respectively, as shown in Figure 5(b).

Table 2 shows a comparison made between the simulation results of our recent work and previous results of [8-10]. The gain and bandwidth are the highest. The antenna proposed in [8] provides the wider bandwidth compared to our proposed antenna. However, the gain of our proposed antenna is higher than that of [8].
TABLE 2: Comparison with previous results.

\begin{tabular}{lcccc}
\hline Refs. & {$[8]$} & {$[9]$} & {$[10]$} & Recent \\
\hline Gain (dB) & 30 & 8.32 & 5.64 & 24 \\
BW (THz) & 234 & 24.38 & 15.6 & 49.5 \\
\hline
\end{tabular}

Three-dimensional radiation pattern and electric field view are also plotted at lower and upper resonant frequencies, as shown in Figures 6(a) and 6(b), respectively. This mode of proposed patch antenna is similar to TM10 mode generated in conventional microwave nanopatch antenna.

To understand the broadband behavior of the antenna, polar plots (copolar and cross-polar) at both planes $\left(\Phi=90^{\circ}\right.$ and $\Phi=0)$ for different frequencies $(160 \mathrm{THz}$ and $197 \mathrm{THz})$ are plotted in Figures 7(a), 7(b), which depict that proposed inset fed HMIM plasmonic nanopatch antenna behaves identically in the whole band. 


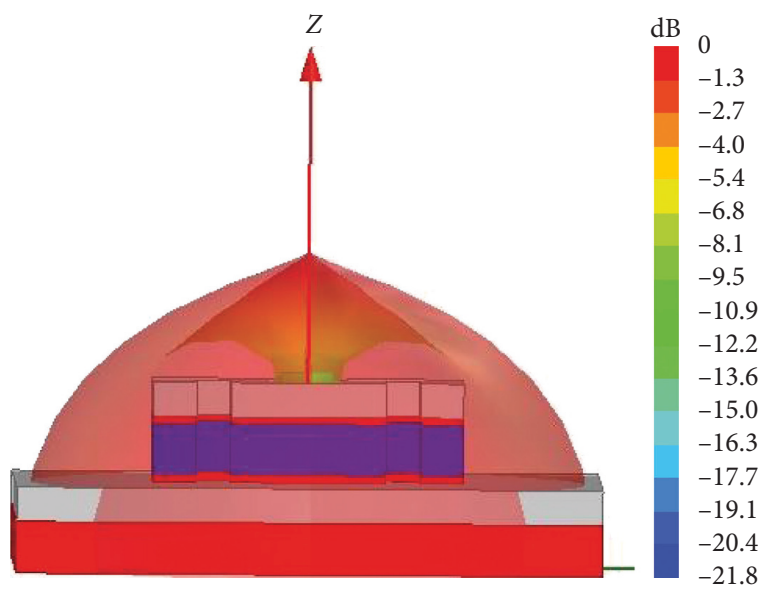

(a)

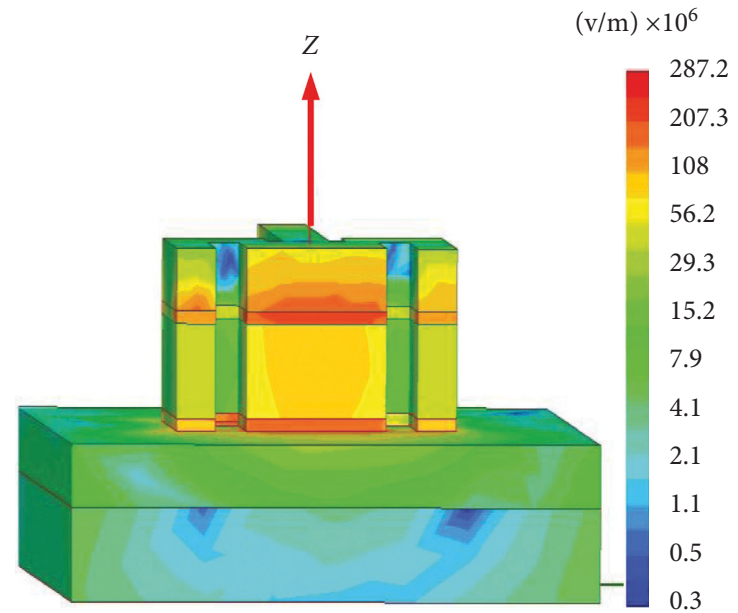

(b)

Figure 6: HMIM nanopatch antenna results for $160.17 \mathrm{THz}$ frequencies. (a) Far-field radiation. (b) Electric field distribution.
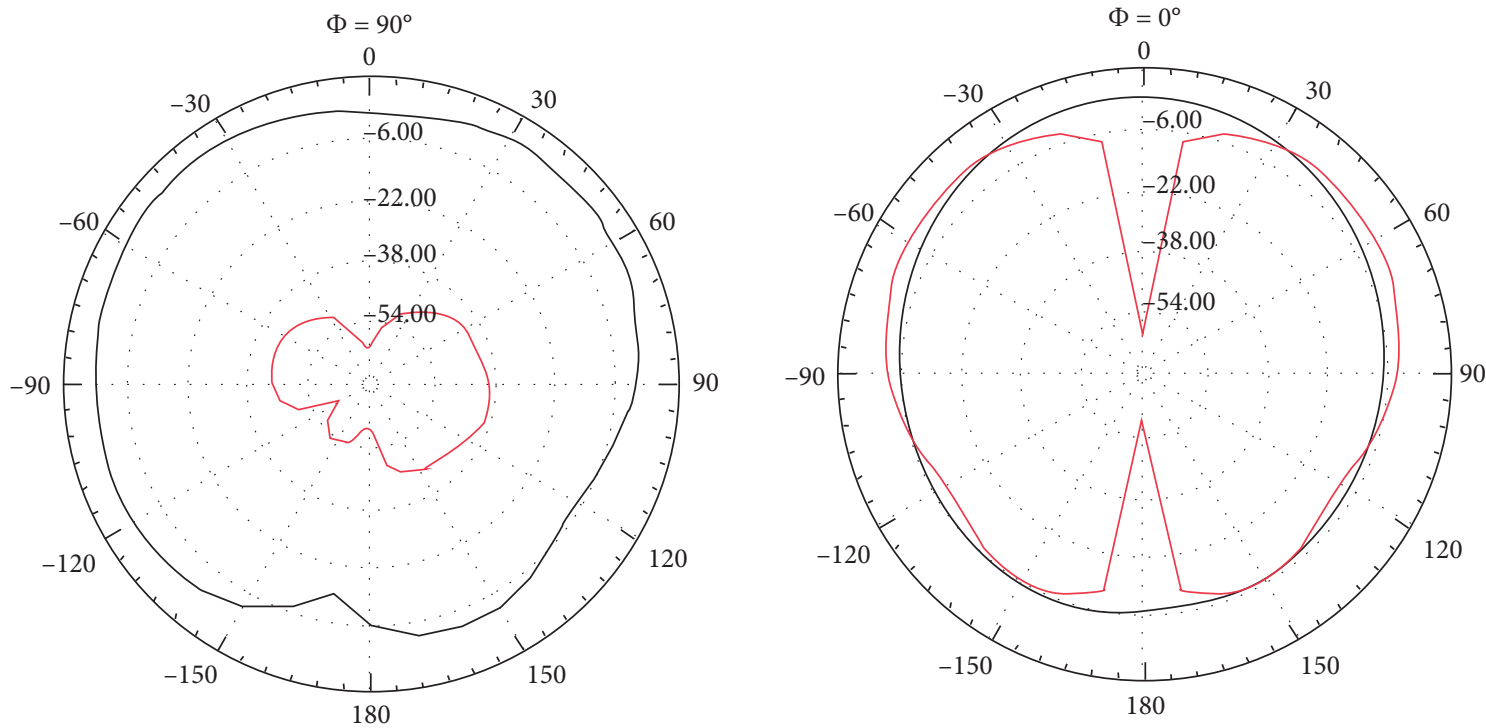

(a)
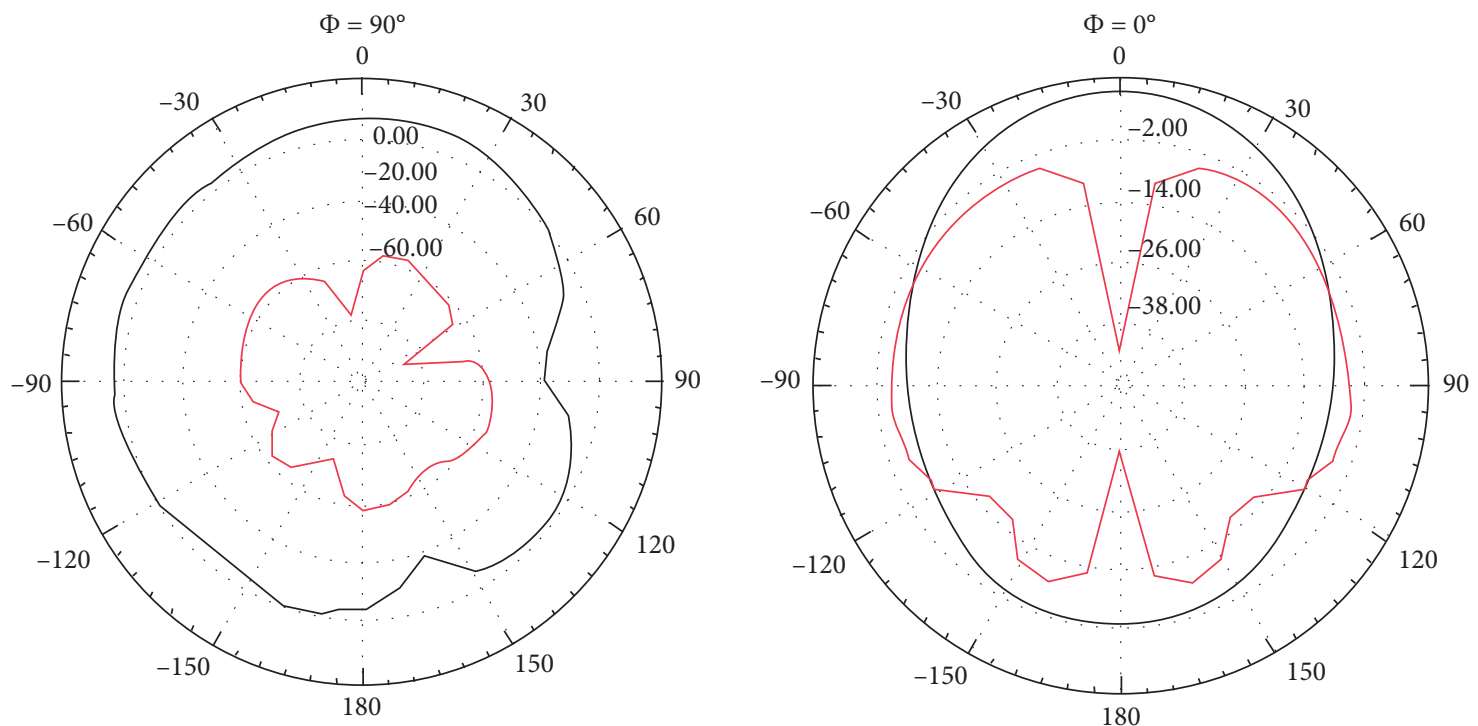

(b)

Figure 7: Polar plots of nanopatch antenna at the frequency of (a) $160 \mathrm{THz}$ and (b) $197 \mathrm{THz}$. Black curves represented copolar and red curves represented cross-polar. 


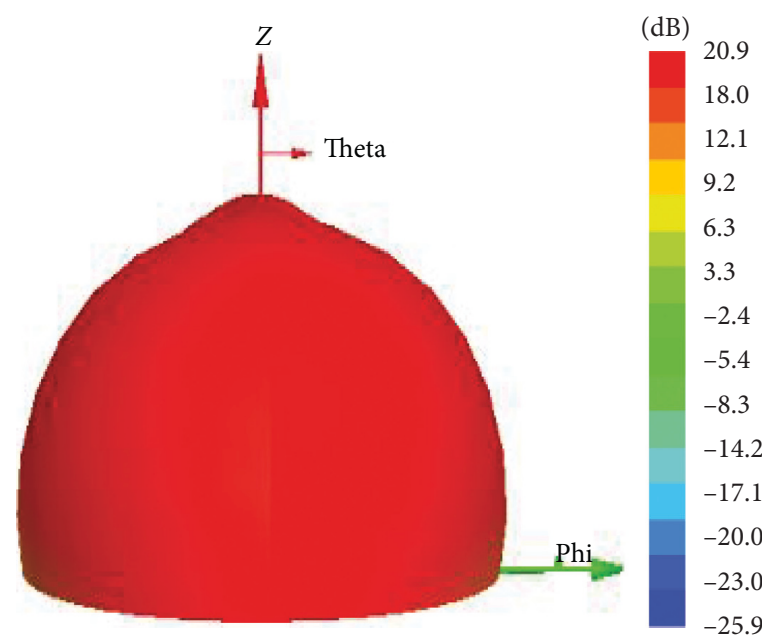

(a)

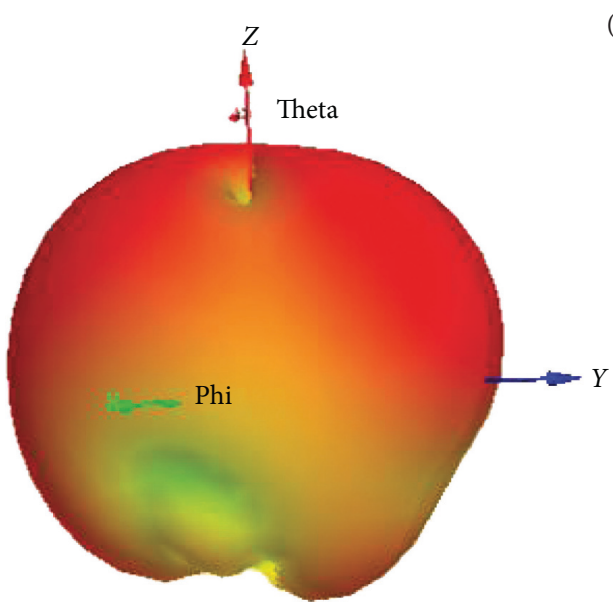

(c)

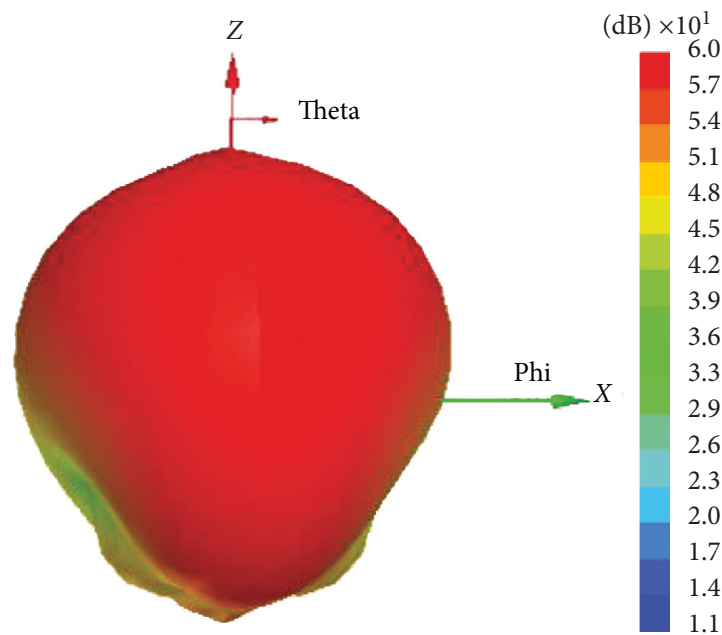

(b)

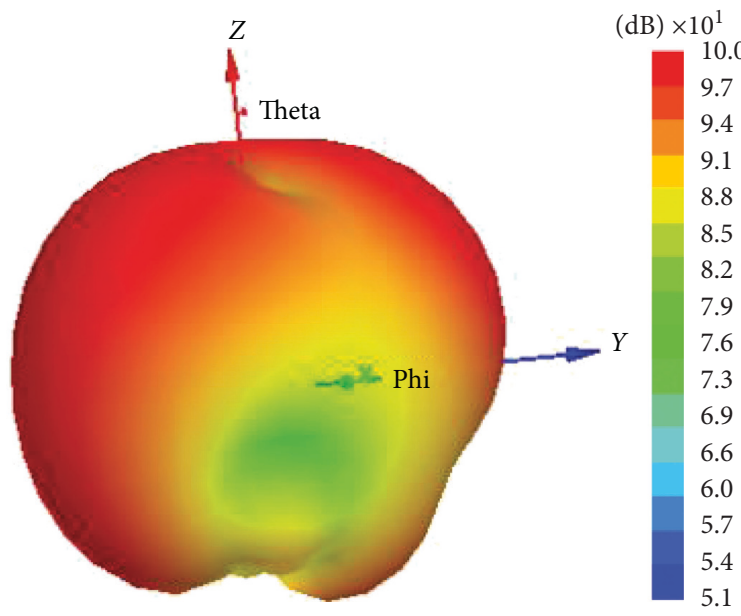

(d)

FIGURE 8: 3D radiation pattern of nanoantenna array at lower and upper resonant frequencies 160 and $197 \mathrm{THz}$.

Three-dimensional radiation pattern for linear array of elements in $x(100 \times 1), y(1 \times 100)$, and $x y(10 \times 10)$ directions is shown in Figures $8(\mathrm{a})-8(\mathrm{~d})$, which clearly depict that there is a remarkable improvement in gain and it can be improved further by increasing the number of elements.

\section{Conclusion}

A realistic waveguide-fed nanopatch antenna based on hybrid metal-insulator-metal (HMIM) plasmonic waveguide is proposed and developed for operation frequency of $125 \mathrm{THz}-250 \mathrm{THz}$. The calculations of impedances of the antenna and the plasmonic waveguide feed are obtained by using Ansoft High Frequency Structure Simulator (HFSS) based on the finite element method (FEM). The proposed antenna is showing more advantages compared to previously reported plasmonic antennas. Antenna arrays are also designed for high-gain applications. This work may be advantageous for several nanophotonics applications like wireless interconnects, energy harvesting, and beam steering.

\section{Data Availability}

The data used to support the findings of this study have been deposited in the HFSS repository.

\section{Conflicts of Interest}

The authors declare that they have no conflicts of interest.

\section{References}

[1] S. M. Sadeghi, R. R. Gutha, and H. Ali, "Super-plasmonic cavity resonances in arrays of flat metallic nanoantennas," Journal of Optics, vol. 21, Article ID 035001, 2019.

[2] Q. Ding and K. C. Toussaint Jr., "Relaying of the local enhanced electric-field using stacked gold bowtie nanoantennas," Nanotechnology, vol. 30, no. 36, Article ID 365202, 2019.

[3] M. A. Basyooni, A. M. Ahmed, and M. Shaban, "Plasmonic hybridization between two metallic nanorods," Optik, vol. 172, 2018. 
[4] M. Chen, L. Chang, T. Xiuqian, X. Xiao, and H. Chen, "Plasmonic quarter-wave plate with $U$-shaped nanopatches," Optik, vol. 134, pp. 179-186, 2017.

[5] T. B. Hoang, J. Huang, and M. H. Mikkelsen, "Colloidal synthesis of nanopatch antennas for applications in plasmonics and nano photonics," Journal of Visualized Experiments, vol. 111, p. 53876, 2016.

[6] T. Shegai, S. Chen, V. D. Miljković, G. Zengin, P. Johansson, and M. Käll, "A bimetallic nanoantenna for directional colour routing," Nature Communications, vol. 481, no. 2, 2011.

[7] D. Ramaccia, F. Bilotti, A. Toscano, and A. Massaro, "Efficient and wideband horn nanoantenna," Optics Letters, vol. 36, no. 10, pp. 1743-1745, 2011.

[8] L. Yousefi and A. C. Foster, "Waveguide-fed optical hybrid plasmonic patch nano-antenna," Optics Express, vol. 20, pp. 18326-18335, 2012.

[9] M. Saad-Bin-Alam, M. I. Khalil, A. Rahman, and A. M. Chowdhury, "Hybrid plasmonic waveguide fed broadband nanoantenna for nanophotonic applications," IEEE Photonics Technology Letters, vol. 27, no. 10, pp. 10921095, 2015.

[10] P. Sharma and V. Dinesh Kumar, "Multilayer hybrid plasmonic nano patch antenna," Plasmonics, vol. 14, no. 2, pp. 435-440, 2018.

[11] T. Shegai, V. D. Miljkovic, K. Bao et al., "Unidirectional broadband light emission from supported plasmonic nanowires," Nano Letters, vol. 706, no. 11, 2011.

[12] H. A. Atwater and A. Polman, "Plasmonics for improved photovoltaic devices," Nature Materials, vol. 9, no. 3, 2015.

[13] N. Yu, P. Genevet, M. A. Kats et al., "Light propagation with phase discontinuities: generalized laws of reflection and refraction," Science, vol. 334, no. 6054, pp. 333-337, 2011.

[14] D. M. Solís, J. M. Taboada, F. Obelleiro, and L. Landesa, "Optimization of an optical wireless nanolink using directive nanoantennas," Optics Express, vol. 2369, no. 21, 2013.

[15] L. Novotny and S. J. Stranick, "Near-field optical microscopy and spectroscopy with pointed probes," Annual Review of Physical Chemistry, vol. 57, no. 1, pp. 303-331, 2006.

[16] J. N. Anker, W. P. Hall, O. Lyandres, N. C. Shah, J. Zhao, and R. P. Van Duyne, "Biosensing with plasmonic nanosensors," Nature Materials, vol. 7, pp. 308-319, 2008.

[17] J. A. Schuller, T. Taubner, and M. L. Brongersma, "Optical antenna thermal emitters," Nature Photonics, vol. 658, no. 3, 2009.

[18] T. W. Ebbesen, H. J. Lezec, H. F. Ghaemi, T. Thio, and P. A. Wolff, "Extraordinary optical transmission through subwavelength hole arrays," Nature London, vol. 391, pp. 668667, 1998.

[19] D. K. Gramotnev and S. I. Bozhevolnyi, "Plasmonics beyond the diffraction limit," Nature Photonics, vol. 4, no. 2, pp. 83-91, 2010.

[20] R. Zia, J. A. Schuller, A. Chandran, and M. L. Brongersma, "Plasmonics: the next chip-scale technology," Materials Today, vol. 7-8, no. 9, pp. 20-27, 2006.

[21] H. Raether, Surface Plasmons on Smooth Surfaces, Springer, Berlin, Germany, 1998.

[22] S. I. Bozhevolnyi, V. S. Volkov, E. Devaux, J. Y. Laluet, and T. W. Ebbesen, "Channel plasmon subwavelength waveguide components including interferometers and ring resonators," Nature, vol. 440, p. 7083, 2006.

[23] E. Verhagen, M. Spasenović, A. Polman, and L. K. Kuipers, "Nanowire plasmon excitation by adiabatic mode transformation," Physical Review Letters, vol. 102, Article ID 203904, 2009.
[24] C. Delacour, S. Blaize, P. Grosse et al., "Efficient directional coupling between silicon and copper plasmonic nanoslot waveguides: toward Metal-Oxide-Silicon nanophotonics," Nano Letters, vol. 10, no. 8, pp. 2922-2926, 2010.

[25] R. F. Oulton, V. J. Sorger, D. A. Genov, D. F. P. Pile, and $\mathrm{X}$. Zhang, "A hybrid plasmonic waveguide for subwavelength confinement and long-range propagation," Nature Photonics, vol. 2, no. 8, pp. 496-500, 2008.

[26] A. Baron, E. Devaux, J.-C. Rodier et al., "Compact antenna for efficient and unidirectional launching and decoupling of surface plasmons," Nano Letters, vol. 11, no. 10, pp. 42074212, 2011.

[27] D. Dregely, R. Taubert, J. Dorfmüller, R. Vogelgesang, K. Kern, and H. Giessen, "3D optical Yagi-Uda nanoantenna array,” Nature Communications, vol. 267, no. 2, 2011.

[28] J. Dorfmuller, R. Vogelgesang, W. Khunsin, C. Rockstuhl, C. Etrich, and K. Kern, "Plasmonic nanowire antennas: experiment, simulation, and theory," Nano Letters, vol. 9, no. 10, pp. 3596-3603, 2010.

[29] Z. Fang, L. Fan, C. Lin, D. Zhang, A. J. Meixner, and X. Zhu, "Plasmonic coupling of bow tie antennas with Ag nanowire," Nano Letters, vol. 11, no. 4, pp. 1676-1680, 2011.

[30] K. J. A. Ooi, P. Bai, M. X. Gu, and L. K. Ang, "Design of a monopole-antenna-based resonant nanocavity for detection of optical power from hybrid plasmonic waveguides," Optics Express, vol. 19, no. 18, pp. 17075-17085, 2011.

[31] N. Kashyap, Z. A. Wani, R. Jain, and V. D. Kumar, "Investigation of a nanostrip patch antenna in optical frequencies," Applied Physics A: Materials Science and Processing, vol. 117, no. 2, pp. 725-729, 2014.

[32] L. Yousefi, "Highly directive hybrid plasmonic leaky wave optical nano-antenna," Progress In Electromagnetics Research Letters, vol. 50, no. 50, pp. 85-90, 2014.

[33] G. N. Malheiros-Silveira, G. S. Wiederhecker, and H. E. Hernández-Figueroa, "Dielectric resonator antenna for applications in nanophotonics," Optic Express, vol. 1, no. 21, pp. 1234-1239, 2013.

[34] P. B. Johnson and R. W. Christy, "Optical constants of the noble metals," Physical Review B, vol. 6, no. 12, pp. 4370-4379, 1972. 


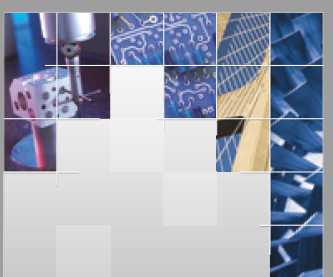

\section{Enfincering}
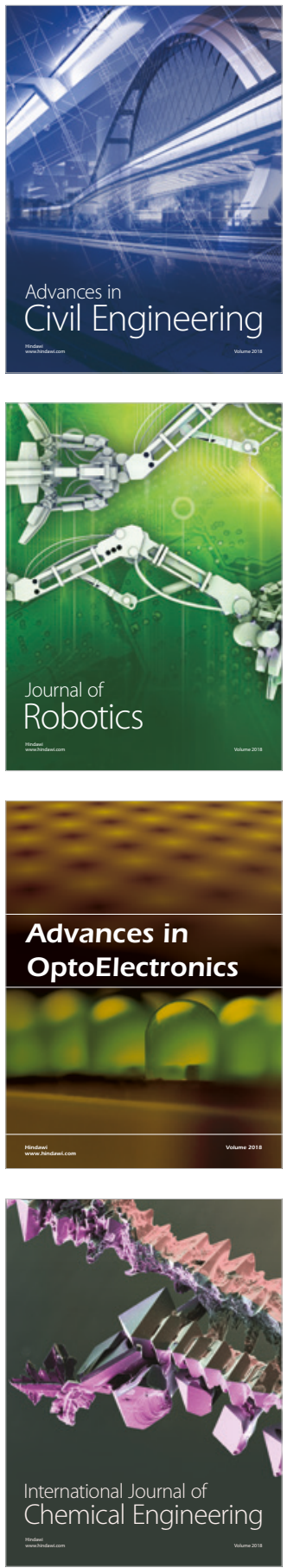

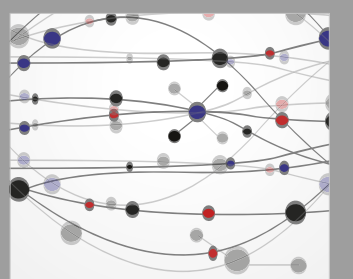

\section{Rotating \\ Machinery}

The Scientific World Journal

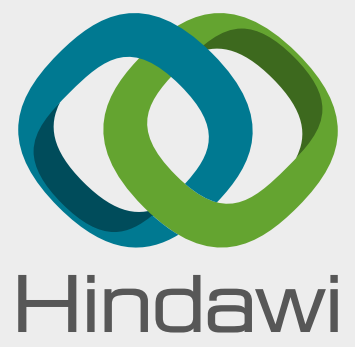

Submit your manuscripts at

www.hindawi.com
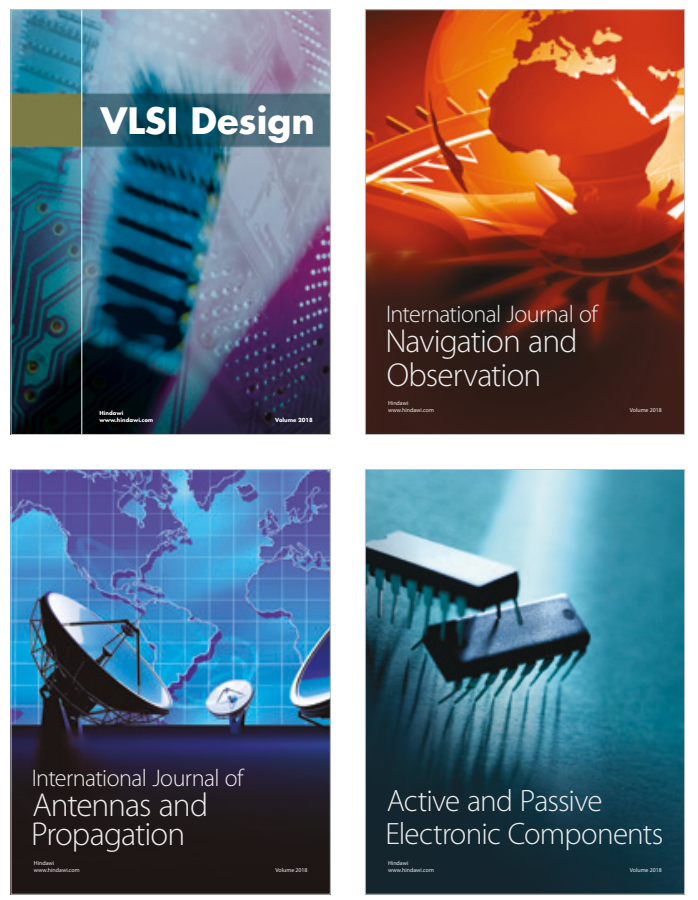
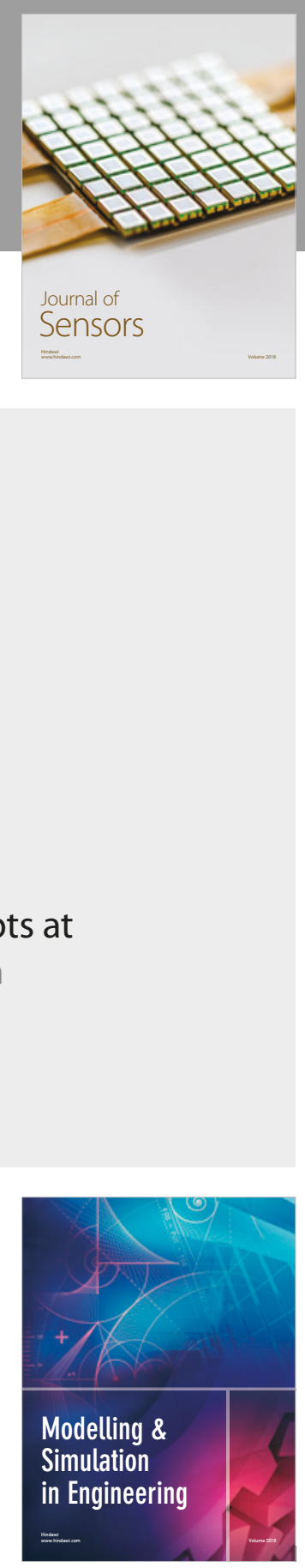

\section{Advances \\ Multimedia}
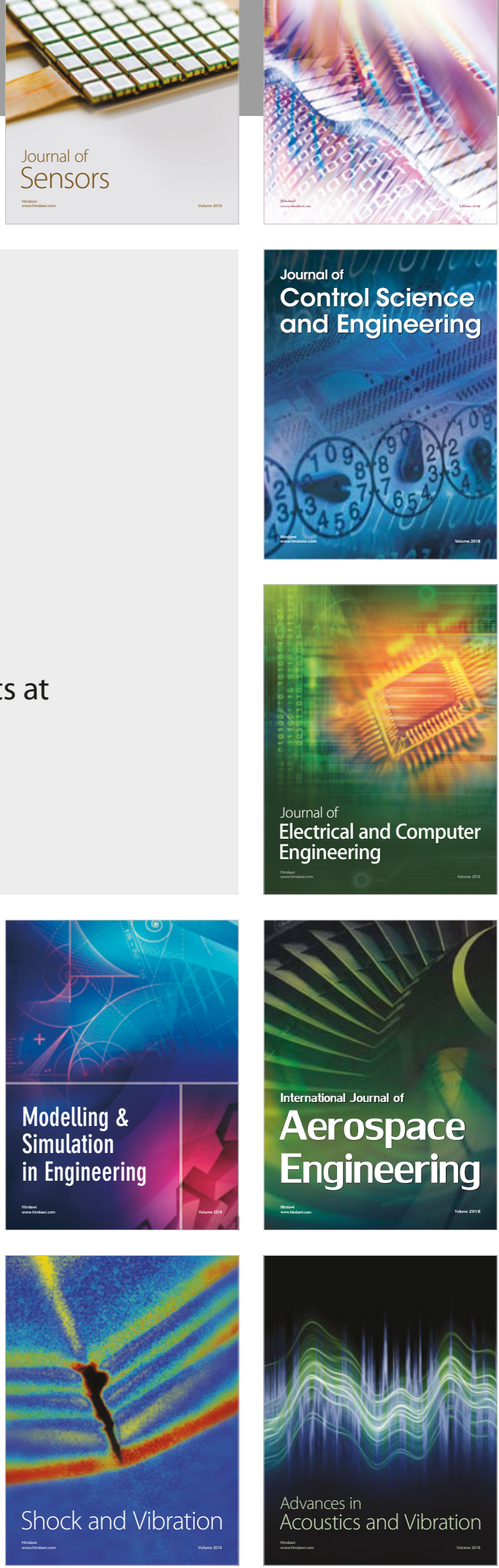